\title{
Eficiencia de la respuesta superovulatoria del ganado Brahman al protocolo P-24
}

\section{Efficiency of superovulatory response to P-24 protocol in fixed time embryo transfer in Brahman cattle}

\author{
Roger Salgado O, ${ }^{1 *}$ M.Sc, Andrés Mejía A, ${ }^{2}$ MVZ, Pablo Suárez S, ${ }^{2}$ MVZ.
}

\begin{abstract}
${ }^{1}$ Univesidad de Córdoba, Facultad de Medicina Veterinaria y Zootecnia, Departamento de Ciencias Pecuarias A.A 354 Montería, Colombia. ${ }^{2}$ MVZ Ejercicio particular. *Correspondencia: rdsalgado@sinú. unicordoba.edu.co.
\end{abstract}

Recibido: Octubre de 2009; Aceptado: Diciembre de 2010.

\section{RESUMEN}

Objetivo. Evaluar la eficiencia de la respuesta superovulatoria del ganado Brahman al protocolo P-24. Materiales y métodos. Se utilizaron doce vacas Brahman donadoras con más de 60 días postparto, a las cuales se les realizó un total de 21 tratamientos superovulatorios con base en el protocolo P-24. Se realizó la colecta de los embriones a través del método convencional y los embriones fueron clasificados (IETS). Resultados. Se obtuvo un promedio de 9.1 estructuras, 4.4 embriones transferibles, 3.7 embriones congelables y 3.2 embriones degenerados recuperados por colecta. Los estadíos de desarrollo que predominaron fueron mórula $(32.6 \%)$, blastocisto temprano (38\%) y blastocisto $(18.5 \%)$. Se presentó efecto $(p<0.05)$ del número de superovulaciones sucesivas sobre la producción de embriones congelables. Sin embargo, no hubo efecto $(p>0.05)$ sobre las demás variables estudiadas. Se presentó efecto $(p<0.05)$ de la producción promedio de estructuras recuperadas, embriones transferibles y congelables entre las donadoras con una producción mayor o igual a 5, y menor a 5 embriones transferibles. Por el contrario, no hubo efecto $(p>0.05)$ de la producción promedio de embriones degenerados y ovocitos sin fecundar entre ambos grupos. No obstante, la proporción de éstos fue mayor en las donadoras con una menor producción de embriones transferibles (50 y $22.4 \%$ ) vs. las donadoras con mayor producción (39.3 y 14\%) para embriones degenerados y ovocitos sin fecundar respectivamente. Conclusiones. El ganado Brahman tuvo una respuesta superovulatoria eficiente al protocolo P-24 para transferencia de embriones a tiempo fijo.

Palabras clave: Brahman, ganado, superovulación, transferencia de embriones. (Fuente: AIMS). 


\section{ABSTRACT}

Objective. To evaluate the efficiency of superovulatory response to P-24 protocol in fixed time embryo transfer in Brahman cattle. Materials and methods. Twelve Brahman cows donors with more than 60 days postpartum were used. Twenty one cows received a superovulatory treatment based in Protocol P-24. Embryos were collected through the conventional method, and the embryos were classified (IETS). Results. An average of 9.1 embryos were collected, 4.4 were considered for direct transfer, 3.7 were considered for freezing and 3.2 were classified as degenerated. According to the stage embryos were classified as: morulae (32.6\%), early blastocyst (38\%) and blastocyst (18.5\%). Effect was presented $(p<0.05)$ in the number of successive superovulations on the production of frozen embryos; however, there was no effect $(p>0.05)$ on the other variables studied. Effect was presented $(p<0.05)$ in the average production of structures recovered, and transferable and frozen embryos among the donors with an output greater than or equal to 5 , and less than 5 transferable embryos. By contrast, there was no effect $(p>0.05)$ on the average production of degenerate embryos and unfertilised oocytes among both groups. Nevertheless, the proportion of these ones is greater in the donors with a lower production of transferable embryos ( 50 and $22.4 \%$ ) vs. donor with the highest production (39.3 and $14 \%)$ for degenerated embryos and unfertilised oocytes respectively. Conclusions. The Brahman cattle has an efficient superovulatory response to P-24 protocol for fixed time embryo transfer.

Key words: Brahman, cattle, embryo transfer, superovulation. (Source: AIMS).

\section{INTRODUCCIÓN}

En Colombia y en Latinoamérica existe una creciente demanda para multiplicar animales de alto valor genético, de razas Bos taurus indicus por su capacidad de adaptación y de producción bajo condiciones climáticas de trópico bajo. La transferencia de embriones está siendo cada vez, la técnica más utilizada en todo el mundo para aprovechar el potencial genético de animales superiores (1). Esta técnica consiste en inducir ovulaciones múltiples mediante tratamientos hormonales en hembras donantes, que son inseminadas y siete días después son colectados los embriones, los cuales pueden ser congelados o transferidos en fresco a hembras receptoras que sirven de madres sustitutas.

El éxito de un programa de transferencia de embriones se mide por el número de terneros que nacen vivos por hembra donante en un determinado lapso de tiempo (2). Una de las limitantes de la transferencia de embriones es la variación existente en la respuesta súper estimulante en bovinos (3).
La sincronía de la ovulación es uno de los factores reportados como causa de pobre respuesta superovulatoria (2).

Existen algunos estudios en donde se han utilizado inductores de ovulación para asegurar un pico preovulatorio de $\mathrm{LH}$ en vacas superovuladas y se ha realizado el retraso del retiro del dispositivo intravaginal de progesterona, en el cual la fuente de progesterona se mantiene hasta 24 ó 36 horas después de la aplicación de PGF2a, con el propósito de que los folículos mas pequeños tengan mayor tiempo para adquirir la capacidad ovulatoria, y mejorar así la sincronía de la ovulación en las donadoras (1-3). Estos trabajos han sido realizados principalmente en hembras de raza Nelore, necesitando evaluar su efecto en las hembras de raza Brahman (1).

El objetivo de este trabajo fue evaluar la eficiencia en la respuesta superovulatoria en bovinos de la raza Brahman al protocolo P-24. 


\section{MATERIALES Y MÉTODOS}

Tipo de estudio. Se realizó un estudio de tipo experimental, descriptivo y prospectivo, el cual se llevó a cabo entre los meses de Mayo y Octubre de 2008.

Sitio de estudio. Se realizó en la granja de la Facultad de Medicina Veterinaria y Zootecnia de la Universidad de Córdoba, ubicada en el corregimiento de Berástegui, Municipio de Ciénaga de Oro, departamento de Córdoba. La zona corresponde a bosque húmedo tropical, altitud de $10 \mathrm{msnm}$, temperatura promedio de $32^{\circ} \mathrm{C}$, humedad relativa de $84 \%$ y precipitación anual de $1100 \mathrm{~mm}$.

\section{Unidades experimentales y normas} de manejo. Se utilizaron 12 vacas Brahman comercial, cíclicas, multíparas; con edades entre 6 y 7 años; de más de 60 días posparto; con un puntaje de condición corporal de 3.5 (escala de 1 a 5 ) obedeciendo al criterio adoptado por Parker (4); y que se encontraban aptas reproductivamente para programas de transferencia de embriones (Clínicamente sanas, libres de enfermedades reproductivas, adecuado estado anatómico y fisiológico del tracto reproductivo en general). Los animales fueron manejados en condiciones de pastoreo, en potreros con oferta forrajera de Angleton (Dichantium aristatum), disposición permanente de agua y sal mineralizada a voluntad, fueron previamente desparasitadas y se les aplicó vitaminas y minerales (Calfosvit Se California ${ }^{\circledR}, 20 \mathrm{~cm}$ por vía intramuscular durante 3 días), antes de comenzar la superovulación. Todos los animales recibieron el protocolo hormonal P-24, diseñado por Zanenga et al (5):

Día 0. Aplicación de un dispositivo intravaginal de progesterona (DIBSintex( $)$ ), más $2.5 \mathrm{mg}$ de benzoato de estradiol (Sintex $($ ) y $50 \mathrm{mg}$ de progesterona (Gestavec, Vecol $®$ ), ambos por vía intramuscular. Día 4: Inicio del tratamiento superovulatorio utilizando una dosis total de $240 \mathrm{mg}$ de FSH (Foltropin-V, Bioniche $\AA$ ), con un esquema de ocho inyecciones por vía intramuscular
(6:00 a.m. y 6:00 p.m.) dosificadas en forma decreciente $(40 \%, 30 \%, 20 \%$ y $10 \%$ de la dosis total). Día 6: Aplicación intramuscular de dos inyecciones de 150

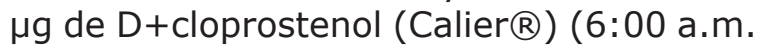
y 6:00 p.m.). Día 7: Retiro del DIB por la mañana (6:00 a.m.). Día 8: Aplicación intravenosa de $0.25 \mathrm{mg}$ de GnRH (Fertagyl, Intervet $\AA)$ por la mañana (6:00 a.m.) e inseminación artificial a tiempo fijo a las 12 y 24 horas después de la aplicación de la GnRH (Día 8: 6 p.m. y Día 9: 6 a.m.) con semen congelado de probada calidad del mismo toro y del mismo lote.

Las donadoras se superovularon en grupos de 3 , aquellas vacas que fueron sometidas al experimento para obtener embriones más de una vez, tuvieron un periodo de descanso de 2 meses entre episodios de superovulación, para un máximo de 3 tratamientos superovulatorios por vaca.

De las 12 donadoras que se manejaron en el experimento, 12 fueron superovuladas por primera vez, 6 por segunda vez y 3 por tercera vez, para un total de 21 tratamientos de superovulación.

Colecta, clasificación y calificación de embriones. El día 15 por la mañana (7 días después de aplicado el inductor de ovulación) se realizó la colecta de los embrionesa través del método convencional, en donde se evaluó la respuesta ovárica al tratamiento superovulatorio con ayuda de un ecógrafo, dotado de un transductor lineal de 7.5 Mhz; se realizó la anestesia epidural de la donante y luego se introdujo y se fijó un catéter armado a partir de una sonda de Foley en la curvatura mayor de cada cuerno uterino (6). Esta sonda a su vez se conectó a un circuito cerrado de 2 vías, a través de una de ellas, fue introducido el medio de lavado (PBS $+1 \%$ de Suero Fetal Bovino) de forma interrumpida, en cantidades de 30 a $50 \mathrm{ml}$ hasta completar $500 \mathrm{ml}$ por cada cuerno; y a través de la otra se recogió el medio con los embriones en un filtro plástico en donde se colectaron los embriones (6). El volumen de la solución que contuvo los embriones fue depositado en placas de Petri en donde se realizó la búsqueda, clasificación y calificación de 
los embriones según las normas de la Sociedad Internacional de Transferencia de Embriones (IETS)(7).

Determinación del número de estructuras recuperadas, embriones transferibles y congelables por colecta. La búsqueda y clasificación de los embriones de acuerdo a su estadio, se realizó en el Laboratorio de Reproducción Animal Tropical (LABRA) de la Facultad de Medicina Veterinaria y Zootecnia de la Universidad de Córdoba, y se determinó por medio de un estereoscopio Nikon SMZ645 y aumentos utilizados de $100 \mathrm{x}$, el número total de estructuras recuperadas y el número de embriones transferibles, congelables y degenerados por colecta.

Respuesta a los tratamientos de superovulación sucesivos. Para efectos de evaluar las respuestas a los tratamientos de superovulación sucesivos realizados, se agruparon las respuestas de los animales superovulados por primera, segunda y tercera vez.

\section{Respuesta superovulatoria de donadoras con una producción promedio mayor o igual a 5, y menor a 5 embriones transferibles. Se agruparon las vacas de acuerdo con la producción promedio de embriones transferibles (mayor o igual a 5 y menor a 5), durante los 21 lavados realizados, con el fin de comparar la respuesta superovulatorias en ambos grupos.}

Análisis estadístico. La determinación del número de estructuras recuperadas, estadio de desarrollo embrionario, número de embriones transferibles, congelables y degenerados del total de colectas, se realizó a través de estadística descriptiva.

La evaluación del efecto que ejerce el número de superovulaciones sobre la respuesta ovárica se realizó a través de un análisis de varianza (ANAVA) y se establecieron diferencias significativas por medio de la prueba de Duncan.

La evaluación de la respuesta superovulatoria de las donadoras con una producción promedio mayor o igual, y menor a 5 embriones transferibles se realizó a través de una prueba T de Student.

\section{RESULTADOS}

Número de estructuras, embriones transferibles, congelables y degenerados recuperados por colecta de las donadoras. Los resultados de la tabla 1 muestran que el número de estructuras, embriones transferibles, congelables y degenerados recuperados por colecta fue de: $9.1,4.4,3.7$ y 3.2 respectivamente.

Tabla 1. Número de estructuras, embriones transferibles, congelables y degenerados (promedio \pm desviación) del protocolo P24 en donadoras Brahman.

\begin{tabular}{lc}
\hline & $\begin{array}{c}\text { P-24 en donadoras } \\
\text { Brahman* }\end{array}$ \\
\hline Número de animales & 12 \\
Número de Estructuras & $9.14 \pm 5.62$ \\
Embriones Transferibles & $4.4 \pm 3.87$ \\
Embriones Congelables & $3.71 \pm 3.85$ \\
Embriones Degenerados & $3.19 \pm 3.09$ \\
\hline
\end{tabular}

* Los resultados expuestos corresponden al promedio de un total de 21 réplicas.

Estadio de desarrollo de los embriones. En la figura 1 se muestra el porcentaje encontrado para cada uno de los estadios de desarrollo embrionario obtenidos.

Los resultados muestran que los estadios de desarrollo embrionario predominantes

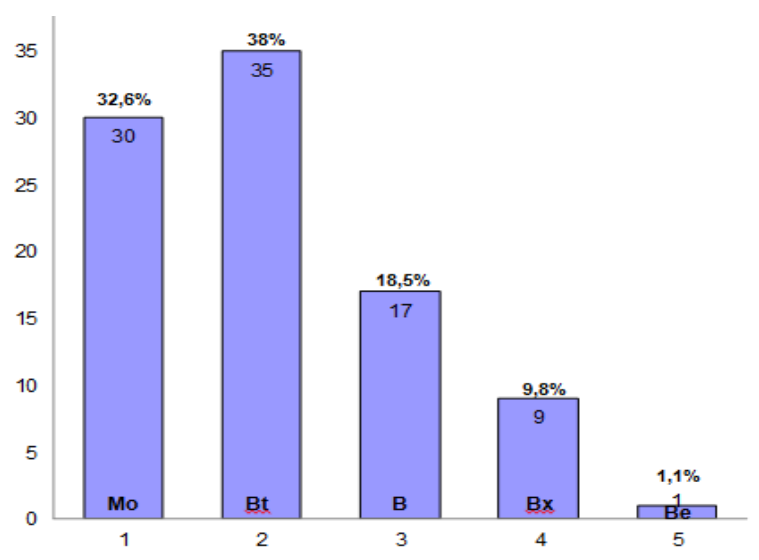

Figura 1. Estadios de desarrollo embrionario obtenidos con el protocolo P-24. 
fueron los estadios de mórula, blastocisto temprano y blastocisto con $32.6,38$ y $18.5 \%$ respectivamente. En menor grado se encontraron los estadios de blastocisto expandido y eclosionado, con 9.8 y $1.1 \%$ respectivamente.

Número de superovulaciones $\left(1^{\circ}, 2^{\circ}\right.$ y $3^{\circ}$ repetición) y su efecto sobre la respuesta superovulatoria en ganado Brahman. Se presentó efecto $(p<0.05)$ del número de superovulaciones sobre la producción de embriones congelables en los tratamientos sucesivos, (Tabla 2). Sin embargo, no hubo efecto $(p>0.05)$ del número de tratamientos sucesivos sobre las demás variables que hacen parte de la respuesta superovulatoria (embriones transferibles, degenerados y ovocitos sin fertilizar).

Tabla 2. Efecto del número de tratamientos superovulatorios sucesivos sobre la respuesta superovulatoria en donadoras Brahman.

\begin{tabular}{lcccc}
\hline & $\begin{array}{c}\text { Donadoras 1a } \\
\text { Superovula- } \\
\text { ción* }\end{array}$ & $\begin{array}{c}\text { Donadoras 2 } \\
\text { Superovula- } \\
\text { ción** }\end{array}$ & $\begin{array}{c}\text { Donadoras 3a } \\
\text { Superovula- } \\
\text { ción*** }\end{array}$ & $\begin{array}{c}\text { Valor } \\
\text { de P }\end{array}$ \\
\hline $\begin{array}{l}\mathrm{N}^{0} \text { de } \\
\text { Estructuras }\end{array}$ & $8.91 \pm 6.08^{\mathrm{a}}$ & $8.66 \pm 4.80^{\mathrm{a}}$ & $11.07 \pm 7.0^{\mathrm{a}}$ & 0.838 \\
$\begin{array}{l}\text { Embriones } \\
\text { Transferibles }\end{array}$ & $5.58 \pm 4.46^{\mathrm{a}}$ & $2.66 \pm 1.75^{\mathrm{a}}$ & $3.0 \pm 3.6^{\mathrm{a}}$ & 0.163 \\
$\begin{array}{l}\text { Embriones } \\
\text { Congelables }\end{array}$ & $5.33 \pm 4.2^{\mathrm{a}}$ & $2.16 \pm 1.60^{\mathrm{b}}$ & $0.33 \pm 0.57^{\mathrm{bc}}$ & 0.034 \\
$\begin{array}{l}\text { Embriones } \\
\text { Degenerados }\end{array}$ & $2.0 \pm 2.17^{\mathrm{a}}$ & $4.16 \pm 3.31^{\mathrm{a}}$ & $6.0 \pm 4.3^{\mathrm{a}}$ & 0.575 \\
$\begin{array}{l}\text { Ovocitos sin } \\
\text { Fertilizar }\end{array}$ & $1.33 \pm 2.3^{\mathrm{a}}$ & $1.83 \pm 2.4^{\mathrm{a}}$ & $2.0 \pm 2.64^{\mathrm{a}}$ & 0.768 \\
\hline
\end{tabular}

Letras diferentes en una misma fila indican diferencia estadística $(p<0.05)$. * Los resultados son de un total de 12 réplicas. **Los resultados son de un total de 6 réplicas. $* * *$ Los resultados son de un total de 3 réplicas

\footnotetext{
Comparación entre la respuesta superovulatoria de donadoras con una producción promedio mayor o igual a 5, y menor a 5 embriones transferibles en los tratamientos superovulatorios realizados. Se presentó efecto $(p<0.05)$ entre el promedio de estructuras y el promedio de embriones transferibles y congelables colectados por donadora de ambos grupos, tal como se observa en la tabla 3. Sin embargo, no hubo efecto ( $p>0.05$ ) entre el número de embriones degenerados y el número de ovocitos sin fertilizar en ambos grupos.
}

Tabla 3. Respuesta superovulatoria de donadoras con una producción promedio mayor o igual a 5, y menor a 5 embriones transferibles.

\begin{tabular}{lcc}
\hline & $\begin{array}{c}\text { Donadoras con } \\
\text { producción } \geq \mathbf{a} 5 \\
\text { embriones } \\
\text { transferibles }\end{array}$ & $\begin{array}{c}\text { Donadoras con } \\
\text { producción < a 5 } \\
\text { embriones } \\
\text { transferibles }\end{array}$ \\
\hline $\begin{array}{l}\text { No animales } \\
\text { No Estructuras por } \\
\text { donadora }\end{array}$ & 5 & 7 \\
$\begin{array}{l}\text { Embriones transferibles } \\
\text { por donadora }\end{array}$ & $12.93 \pm 5.05^{\mathrm{a}}$ & $5.19 \pm 3.32^{\mathrm{b}}$ \\
$\begin{array}{l}\text { Embriones congelables } \\
\text { por donadora }\end{array}$ & $6.0 \pm 2.85^{\mathrm{a}}$ & $1.95 \pm 1.30^{\mathrm{b}}$ \\
$\begin{array}{l}\text { Embriones degenerados } \\
\text { por donadora }\end{array}$ & $4.03 \pm 2.47^{\mathrm{a}}$ & $1.88 \pm 1.17^{\mathrm{b}}$ \\
$\begin{array}{l}\text { Ovocitos sin fertilizar } \\
\text { Letras diferentes en una misma }\end{array}$ & $2.60 \pm 1.67^{\mathrm{a}}$ & $1.40 \pm 2.96^{\mathrm{a}}$ \\
\hline $\begin{array}{l}\text { Significativamente }(\mathrm{p}<0.05) . \\
\text { difieren }\end{array}$
\end{tabular}

En cuanto al número de embriones degenerados y ovocitos sin fertilizar, no se presentó efecto $(p>0.05)$ de ambos grupos sobre el promedio de éstos (Tabla 4).

Tabla 4. Proporción de embriones degenerados y ovocitos sin fertilizar de donadoras con una producción promedio mayor o igual a 5, y menor a 5 embriones transferibles.

\begin{tabular}{lcc}
\hline & $\begin{array}{c}\text { Donadoras con } \\
\text { producción } \geq \text { a 5 } \\
\text { embriones } \\
\text { transferibles }\end{array}$ & $\begin{array}{c}\text { Donadoras con } \\
\text { producción < a 5 } \\
\text { embriones } \\
\text { transferibles }\end{array}$ \\
\hline $\begin{array}{l}\text { Total estructuras } \\
\text { Total embriones } \\
\text { recuperados }\end{array}$ & 139 & 53 \\
$\begin{array}{l}\text { Proporción } \\
\text { embriones } \\
\text { degenerados (\%) }\end{array}$ & 117 & 42 \\
$\begin{array}{l}\text { Proporción de } \\
\text { ovocitos sin } \\
\text { fertilizar }(\%)\end{array}$ & $46 / 117(39.3)$ & $21 / 42(50)$ \\
\hline
\end{tabular}

\section{DISCUSIón}

El número de estructuras recuperadas por colecta que se obtuvo en el presente estudio fue similar a lo comunicado por Martins et al (8) y Baruselli et al (3) en vacas Nelore con promedios de 7.4 y 10.6 respectivamente; y por Bo et al (9) en vacas y novillas Angus y Brangus con un promedio de 9.1 estructuras recuperadas.

El número de embriones transferibles recuperados por colecta en vacas Brahman, fue similar a lo reportado por Bo et al (9) en 
vacas Angus y Brangus quienes obtuvieron 5.4 embriones transferibles por donadora. De igual manera Martins et al (8) y Baruselli et al (3) obtuvieron promedios similares de 6 y 6.5 embriones transferibles en vacas Nelore.

En cuanto al número de embriones congelables obtenidos por colecta en el presente estudio, resultados similares han sido reportados por Martins et al (8) y Baruselli et al (3) con un promedio de 5.6 y 5.7 respectivamente, en vacas Nelore.

El número de embriones degenerados obtenido en vacas Brahman es similar a lo comunicado por Zanenga et al (5) en vacas Nelore con un promedio de 4.8, sin embargo, Martins et al (8) reportaron un promedio inferior (0.7 embriones degenerados por donadora), en vacas Nelore.

Con relación al estadio de desarrollo, los resultados obtenidos siguen el mismo patrón porcentual a lo reportado por Linder et al, citado por Palma (6), quienes obtuvieron una mayor cantidad de embriones en los estadios de mórula, blastocisto temprano y blastocisto con un 37,30 y $23 \%$ respectivamente, y en menor grado reportaron los estadios de blastocisto expandido con un $6 \%$ y blastocisto eclosionado con un $1 \%$. Resultados similares también han sido comunicados por Kauffold et al citado por Palma (6), en donde predominaron los estadios de mórula, blastocisto temprano y blastocisto con un $32.9,26.4$ y $19.1 \%$ respectivamente, $y$ en menor grado reportaron un $0.9 \%$ de blastocistos expandidos y un $0.4 \%$ de blastocistos eclosionados. Estos resultados demuestran que el protocolo $\mathrm{P}-24$ ejerce un efecto positivo sobre la sincronía de la ovulación, lo cual se ve reflejado en una mayor proporción de embriones con estadios de desarrollo de mórula, blastocisto temprano y blastocisto recolectados, en el día 7 pos inseminación.

Los resultados relacionados con la producción de embriones transferibles obtenidos en el presente trabajo, coinciden con lo reportado por Hasler (10), quien no encontró efecto negativo sobre la producción de embriones transferibles en vacas Angus superovuladas en promedio 13.8 veces; y a lo reportado por Donaldson y Perry, citados por Palma (6), quienes no hallaron variación en la producción de embriones transferibles a lo largo de 10 tratamientos sucesivos. No obstante, Warfield et al, citado por Palma (6), encontraron disminución en la producción de embriones transferibles a partir del tercer tratamiento. Un importante hallazgo en el presente estudio fue que los tratamientos sucesivos disminuyeron significativamente la producción de embriones congelables. Este hecho podría ser atribuido al incremento en la dosis de gonadotropina empleada de un tratamiento a otro; resultado coincidente con lo comunicado por Donaldson (11). Adicionalmente se podría considerar una asincronía en el crecimiento folicular, donde los folículos más maduros serían los primeros en producir grandes cantidades de estrógenos. Por lo tanto, los oocitos dentro de estos folículos estarían expuestos a altas concentraciones de estrógenos por largos periodos antes de la ovulación; esto podría ser la causa de la disminución en la calidad embrionaria (6)

La mayor producción de embriones transferibles se obtuvo a partir de vacas que tuvieron mejor respuesta superovulatoria; a pesar que en el presente estudio no se estudiaron las poblaciones foliculares de los animales, el hecho que existiera un grupo de animales con una mejor respuesta superovulatoria ( $>5$ embriones) podría ser atribuido a la variación en el número de folículos durante las onda, dado que las vacas que presentan gran número de folículos durante las ondas foliculares responden mejor a la superovulación; adicionalmente estas poblaciones foliculares durante la onda tiene una alta repetibilidad (0.84) dentro de individuos (12). Por otra parte, el grupo de vacas con pobre respuesta superovulatoria (< 5 embriones transferibles) tuvo alta repetibilidad, resultados similares han sido reportados por Peixoto et al, citado por Palma (6), quienes encontraron que las respuestas pobres a un primer tratamiento superovulatorio en ganado Bos taurus 
indicus, oscilo entre moderada y alta.

Los resultados obtenidos con el protocolo P-24 en vacas Brahman se encuentran dentro del rango de producción de estructuras recuperadas, embriones transferibles, congelables y degenerados publicados en la literatura reportada. En conclusión estos hallazgos sugieren que el protocolo P-24 es viable para obtener una respuesta superovulatoria eficiente en programas de superovulación y transferencia de embriones a tiempo fijo en donadoras de raza Brahman.

\section{Agradecimientos}

A la Universidad de Córdoba, y al CIUC por la financiación de este proyecto FMV-06-04.

\section{REFERENCIAS}

1. Bo GA, Moreno D, Cutaia L, Baruselli $\mathrm{SP}$, Reis EL. Manipulação hormonal do ciclo estral em donadoras e receptoras de embriao bovino. Acta Sci Vet 2004; 32(suppl):1-22.

2. Bo GA, Baruselli PS, Chesta PM, Martins CM. The timing of ovulation and insemination schedules in superstimulated cattle. Theriogenology 2006; 65(1):89-101

3. Baruselli PS, Marques MO, Reis EL, Nasser LF, Silva RC, Menegatti JA et al. Adequação da dose de FSH (Foltropin-V) em protocolos de superovulação de vacas Nelore (Bos taurus indicus) com inseminação artificial em tempo fixo (SOTF). In: XVII Reuniao anual da sociedade brasileira de tecnología de embrioes; 2003, Fortaleza, Brasil. Acta Sci Vet; 2003b. (31):244-45.

4. Parker R. Using body conditions scoring in dairy herd management. Fact Sheet (Ontario), January 1989.

5. Zanenga CA, Marques MO, Santos IC, Valentin R, Baruselli PS. Comparacao entre dois protocolos de superovulacao com inseminacao artificial em tempo fixo em vacas Nelore (Bos indicus). In: XVII Reuniao anual da sociedade brasileira de tecnología de embrioes; 2003, Fortaleza, Brasil. Acta Sci Vet; 2003.

6. Palma GA. Biotecnología de la reproducción. 2a. Ed. Mar del Plata: Reprobiotec; 2008.
7. Stringfellow $D$, Seidel $S$. Manual of the international embryo transfer society. 3rd edition. IL, USA: Savoy; 1998.

8. Martins CM, Marques MO, Silva RC, Baruselli PS. Adequação do protocolo de superovulação com inseminação artificial em tempo fixo em Bos taurus. [Tesis de Maestría]. Sao Paulo: Universidade de Sao Paulo; 2005.

9. Bo GA, Baruselli PS, Chesta P. Dinámica folicular, momento de la ovulación e inseminación artificial a tiempo fijo en donantes de embriones. En: Jornadas de actualización en biotecnologías de la reproducción en bovinos (IRAC); Córdoba, Argentina; 2006.

10. Hasler JF. Comparation between conventional in vivo and in vitro production of embryos in bovine embryo transfer programs. En: Congreso de Reproducciòn Animal, Rosario, Argentina; 2000.

11. Donalson L. Dose of FSH-p as a source of variation in embryo production from superovulated cows. Theriogenology $1984 ; 22: 205-212$.

12. Ireland JJ, Ward $F$, Jimenez $F$, Ireland $\mathrm{JLH}$, Smith GW, Lonergan P et al. Follicle numbers are highly repeatable within individual animals but are inversely correlated with FSH concentrations and the proportion of good - quality embryos after ovarian stimulation in cattle. Human Reproduction 2007; 22: 1687-95. 